TRANSACTIONS OF THE

AMERICAN MATHEMATICAL SOCIETY

Volume 361, Number 10, October 2009, Pages 5139-5162

S 0002-9947(09)04815-6

Article electronically published on May 19, 2009

\title{
CHEEGER CONSTANTS OF SURFACES AND ISOPERIMETRIC INEQUALITIES
}

\author{
PANOS PAPASOGLU
}

\begin{abstract}
We show that if the isoperimetric profile of a bounded genus noncompact surface grows faster than $\sqrt{t}$, then it grows at least as fast as a linear function. This generalizes a result of Gromov for simply connected surfaces.

We study the isoperimetric problem in dimension 3. We show that if the filling volume function in dimension 2 is Euclidean, while in dimension 3 it is sub-Euclidean and there is a $g$ such that minimizers in dimension 3 have genus at most $g$, then the filling function in dimension 3 is 'almost' linear.
\end{abstract}

\section{INTRODUCTION}

If $M$ is a Riemannian manifold of dimension $n$, one defines the Cheeger constant $h$ of $M$ by

$$
h(M)=\inf _{A}\left\{\frac{\operatorname{vol}_{n-1}(\partial A)}{\operatorname{vol}_{n}(A)}: \operatorname{vol}_{n}(A) \leq \frac{1}{2} \operatorname{vol}_{n}(M)\right\},
$$

where $A$ ranges over all open subsets of $M$ with smooth boundary. If $M$ is a simplicial manifold, one can similarly define the Cheeger constant of $M$.

As usual we refer to $v l_{2}$ as the area and $v o l_{1}$ as the length. If $M$ is a simplicial 2-manifold or a 2-manifold with a Riemannian metric, we denote by $A(M)$ the area of $M$. Similarly if $p$ is a (simplicial or Riemannian) path, we denote by $l(p)$ the length of $p$.

It follows from the work of Hersch [22] and Yang and Yau [39] (see also [24]) that there is a bound on the Cheeger constant of a closed surface that depends only on its area. So for example there is a constant $c$ such that any Riemannian manifold homeomorphic to the 2 -sphere $S$, which has area 1 , has $h(S) \leq c$. A similar result holds for graphs on bounded genus surfaces by work of Lipton and Tarjan [25] and Gilbert, Hutchinson and Tarjan [15]. In this paper we use these bounds on the Cheeger constants to study isoperimetric profiles of surfaces and higher isoperimetric inequalities.

To make the paper self-contained we give proofs of the Cheeger constant bounds in the first section. Our proofs have the advantage that they are more direct than the existing proofs, as they rely only on the Besicovitch theorem ([2]).

We state these bounds both in the simplicial and in the Riemannian setting. The bounds in the simplicial case are applied in the last section to higher isoperimetric

Received by the editors August 3, 2007.

2000 Mathematics Subject Classification. Primary 53C20, 53C23, $20 \mathrm{~F} 65$.

This work was co-funded by the European Social Fund (75\%) and the National Resources (25\%) (Epeaek II) Pythagoras. 
inequalities. We provide explicit bounds, but the constants below are far from optimal.

Proposition 2.3. Let $S$ be a Riemannian manifold or a simplicial complex homeomorphic to the 2-sphere. Then the Cheeger constant, $h(S)$, of $S$ satisfies the inequality

$$
h(S) \leq \frac{16}{\sqrt{A(S)}},
$$

where $A(S)$ is the area of $S$.

In general we have an upper bound that depends on the genus:

Proposition 2.6. Let $S$ be a closed orientable surface of genus $g \geq 1$ equipped either with a Riemannian metric or with a simplicial complex structure. Let $A(S)$ be its (simplicial or Riemannian) area. Then the Cheeger constant, $h(S)$, of $S$ satisfies the inequality

$$
h(S) \leq \frac{4 \cdot 10^{3} \cdot g^{2}}{\sqrt{A(S)}} .
$$

One easily sees that the same bound applies to surfaces with boundary (just collapse the boundary curves to points to obtain a closed surface). One can also get bounds for non-orientable surfaces by passing to the orientable double cover.

If $\left(M^{n}, g\right)$ is a Riemannian manifold of infinite volume, the isoperimetric profile function of $M^{n}$ is a function $I_{M}: \mathbb{R}^{+} \rightarrow \mathbb{R}^{+}$defined by

$$
I_{M}(t)=\inf _{\Omega}\left\{\operatorname{vol}_{n-1}(\partial \Omega): \Omega \subset M^{n}, \operatorname{vol}_{n}(\Omega)=t\right\},
$$

where $\Omega$ ranges over all regions of $M^{n}$ with smooth boundary. One can similarly define an isoperimetric profile function $I_{M}: \mathbb{N} \rightarrow \mathbb{N}$ for simplicial manifolds $M^{n}$.

Other functions related to the isoperimetric problem are the filling area, $F A_{0}$, and homological filling area, $F A^{h}$, functions of $M$ that we define below. For more information on filling invariants and applications we refer the reader to the seminal paper of Gromov [17.

If $p$ is a smooth contractible closed curve in $M$, we define its filling area, Fill $A_{0}(p)$, as follows: We consider all Riemannian discs $D$, such that there is a 1-Lipschitz map $f: D \rightarrow X$ with $\left.f\right|_{\partial D}=p$. We define $F i l l A_{0}(p)$ to be the infimum of the areas of this collection of disks. We now define the filling area function of $M$ by

$$
F A_{0}(t)=\sup _{p}\left\{F i l l A_{0}(p): l(p) \leq t\right\},
$$

where $p$ ranges over all smooth contractible closed curves of $M$.

More generally we can consider 1-cycles $c$ (i.e. unions of closed curves) that can be filled by 2-cycles to define the homological filling area function (see Section 2 for details).

Gromov (18], ch. 6; see also [10], ch. 6) showed the following:

Gromov's theorem. et $\left(M^{n}, g\right)$ be a simply connected Riemannian manifold. Assume that there is some $t_{0}$ such that for all $t>t_{0}, F A_{0}(t) \leq \frac{1}{16 \pi} t^{2}$. Then there is a constant $K$ such that for all $t>t_{0}, F A_{0}(t) \leq K t$.

Gersten [14] observed that this theorem also holds for homological filling area $F A^{h}$ (see also [18], 6.6E, 6.6F), while Olshanskii [27] gave an elementary proof of Gromov's theorem (see as well [8], [29], [12, for other proofs). 
If the dimension of $M$ is 2 , then there is an obvious link between filling area and isoperimetric profile, so from Gromov's theorem we easily obtain the following:

Corollary. Let $(S, g)$ be a Riemannian manifold homeomorphic to the plane. Assume that there is some $t_{0}$ such that for all $t>t_{0}, I_{S}(t) \geq 4 \sqrt{\pi} \sqrt{t}$. Then there is a constant $\delta>0$ such that for all $t>t_{0}, I_{S}(t) \geq \delta$.

We observe that if we modify the definition of $F A_{0}$ for the plane $S$ so that we allow only simple closed curves, then $F A_{0}$ becomes the inverse function of $I_{S}$. Now it is easy to see that Gromov's theorem applies to this modified $F A_{0}$ as well. So a gap for $F A_{0}$ implies a gap for $I_{S}$.

We note that the isoperimetric problem for surfaces has been studied extensively (see [7, [16, 26, 32, 33], 35]).

We see that the 'gap' in the filling functions implies a 'gap' for the isoperimetric profiles of Riemannian planes. It is reasonable to ask whether there are gaps in the isoperimetric profile of other surfaces. Although this does not hold in general we show that this is true for planes with holes or more generally surfaces of finite genus.

Theorem 3.5. Let $S$ be a plane with holes equipped either with a Riemannian metric or with a simplicial complex structure. Assume that there is some $K>0$ such that for all $t \in[K, 100 K], I_{S}(t) \geq 10^{2} \sqrt{t}$. Then for all $t>K, I_{S}(t) \geq \frac{1}{\sqrt{K}} t$.

One obtains as a corollary that the same holds for finite genus surfaces:

Corollary 3.6. Let $S$ be a complete non-compact surface of finite genus equipped either with a Riemannian metric or with a simplicial complex structure. Assume that there is some $K>0$ such that for all $t \in[K, 100 K], I_{S}(t) \geq 10^{2} \sqrt{t}$. Then there is a constant $\delta>0$ such that for all $t>K, I_{S}(t) \geq \delta t$.

It is an interesting question whether Gromov's theorem on filling area has an analogue for higher dimensional filling functions. The bounds on the Cheeger constants of surfaces can be used to obtain some partial results in this direction. We will state our results in the convenient setting of simplicial complexes. We remark that if $M$ is a compact Riemannian manifold, then the filling functions of its universal covering, $\tilde{M}$, are determined (up to some Lipschitz constant) by $\pi_{1}(M)$ (see 13 , theorems 10.3.3, 10.3.1, and [9]), so one can forget the Riemannian metric and work with a triangulation and simplicial chains to calculate the filling functions of $\tilde{M}$.

To fix ideas when we refer to chains and cycles we always mean chains and cycles for simplicial homology with $\mathbb{Z}$ coefficients (in fact our results apply to $\mathbb{Z}_{2}$ coefficients as well). We denote by $H_{n}(X)$ the $n$th homology group of the space $X$ with $\mathbb{Z}$-coefficients.

Let $X$ be a simplicial complex such that $H_{k}(X)=0$. If

$$
S=\sum n_{i} \sigma_{i}
$$

is a (simplicial) $k$-chain, we define the $k$ th volume of $S$ by $\operatorname{vol}_{k}(S)=\sum\left|n_{i}\right|$. If $S$ is a $k$-cycle, we define the filling volume of $S$ by

$$
\text { Fillvol }_{k+1}(S)=\inf \left\{\operatorname{vol}_{k+1} T: \partial T=S\right\} .
$$

For $k \geq 1$ we define the $(k+1)$ th filling volume function, $F V_{k+1}$, of $X$ by

$$
F V_{k+1}(n)=\sup \left\{F i l l v o l_{k+1}(S): S \text { is a } k \text {-cycle such that } \operatorname{vol}_{k}(S) \leq n\right\} \text {. }
$$


If $S$ is a $k$-cycle such that $\operatorname{vol}_{k}(S) \leq n$ and $\operatorname{Fillvol}_{k+1}(S)=F V_{k+1}(n)$, we say that $S$ is a minimizer for $F V_{k+1}(n)$.

If $S$ is a 2-cycle, one can define the genus of $S$. Indeed $S$ is represented by a map $f: \Sigma \rightarrow X$, where $\Sigma$ is a closed surface and $f$ is simplicial and 1-1 on open 2 -simplices (see [21], sec. 2.1, p. 109). We define the genus of $S$ to be the genus of the surface $\Sigma$.

We remark that $F A^{h}=F V_{2}$. As we noted earlier Gromov's theorem applies to $F A^{h}$ as well, so we have that if

$$
\lim _{n \rightarrow \infty} \frac{F V_{2}(n)}{n^{2}}=0
$$

then there is some $K>0$ such that $F V_{2}(n) \leq K n$ for all $n \in \mathbb{N}$. In general we say that $F V_{k}$ is Euclidean if there is some $K>0$ such that

$$
\frac{1}{K} n^{\frac{k}{k-1}} \leq F V_{k}(n) \leq K n^{\frac{k}{k-1}}, \quad \forall n \in \mathbb{N}
$$

and we say that $F V_{k}$ is sub-Euclidean if

$$
\lim _{n \rightarrow \infty} \frac{F V_{k}(n)}{n^{\frac{k}{k-1}}}=0
$$

So by Gromov's theorem if $F V_{2}$ is sub-Euclidean, then it is linear. We note that a naive guess, that if $F V_{3}$ is sub-Euclidean, then it is linear, is contradicted by Pansu's theorem ([28]), $F V_{3}$ in Heisenberg's group grows like $n^{\frac{4}{3}}$. On the other hand Gromov conjectures ([18, sec. $6 B_{2}$ ) that if $X$ is a $C a t(0)$ space with a co-compact group action, then sub-Euclidean filling implies linear filling in any dimension. More generally it is believed that this is true for spaces satisfying a cone-type inequality (see [38]).

Another possible direction is to examine all filling functions simultaneously. Some specific conjecture is: If $F V_{2}$ is bounded by a quadratic function and $F V_{3}$ is sub-Euclidean, then $F V_{3}$ is bounded by a linear function. Of course one can state this conjecture in any dimension: If $F V_{i}$ is Euclidean for $i=2, \ldots, k-1$ and $F V_{k}$ is sub-Euclidean, then $F V_{k}$ is linear. The following theorem gives some evidence in favor of this conjecture.

Theorem 4.1. Let $X$ be a simplicial complex such that $H_{1}(X)=H_{2}(X)=0$. Assume that the following hold:

- There is some $K>0$ such that $F V_{2}(n) \leq K n^{2}$ for all $n \in \mathbb{N}$.

$\bullet$

$$
\lim _{n \rightarrow \infty} \frac{F V_{3}(n)}{n^{\frac{3}{2}}}=0 .
$$

- There is some $g \in \mathbb{N}$ such that if $S$ is a minimizer 2 - cycle in $X^{2}$, then $S$ is represented by a surface of genus at most $g$.

Then for every $\epsilon>0$ we have

$$
\lim _{n \rightarrow \infty} \frac{F V_{3}(n)}{n^{1+\epsilon}}=0
$$

1.1. Outline of the proofs. The proofs of Proposition 2.3 and Proposition 2.6 are based on the Besicovitch lemma (and more generally co-area inequalities). The idea can be grasped more easily in the case of the sphere. We consider a minimal length simple closed curve $p$ that subdivides the sphere $S$ into two pieces $S_{1}, S_{2}$ such that $A\left(S_{1}\right), A\left(S_{2}\right)$ both have area bigger than $A(S) / 4$. Let's say $A\left(S_{1}\right) \geq A\left(S_{2}\right)$. Now 
$S_{1}$ is a disk and the subpaths of $p$ of length $<l(p) / 2$ are geodesic in $S_{1}$ because $p$ is minimal. Applying the Besicovitch lemma, one sees that $l(p)$ is smaller than $4 \sqrt{A(S)}$. This implies Proposition 2.3 .

To prove Theorem 3.5 we argue by contradiction. We take a 1-cycle $c$ of minimal filling area such that Fill $A(c)>K$ and $l(c)<\frac{1}{\sqrt{K}}$ Fill $A(c)$. If $c=\partial R$, then we collapse the 'holes' of $R$ to points to get a sphere $S$. Applying Proposition 2.3 to $S$ we find a 1-cycle $\gamma$ in $S$ such that $K \leq \operatorname{Fill} A(\gamma) \leq 4 K$ which satisfies the inequality

$$
\operatorname{Fill} A(\gamma)>\frac{l(\gamma)^{2}}{100}
$$

This is somewhat tricky, and the proof also uses Besicovitch's lemma and exploits the convexity of $x^{2}$. Note though that intuitively the existence of such a curve is obvious since $S$ is 'positively curved' at some points. Finally we lift $\gamma$ back to $R$ and we get a cycle with smaller filling area than $c$ that has the same properties as $c$; this contradicts the minimality of $c$. It is an easy corollary that the theorem holds for finite genus surfaces in general.

The proof of Theorem 4.1 is by contradiction. We assume that for some $\epsilon>0$ we have

$$
\lim _{n \rightarrow \infty} \frac{F V_{3}(n)}{n^{1+\epsilon}}=\infty
$$

We take $M$ 'big enough', and we consider a 2-cycle $S$ which is a minimal area counterexample to $F V_{3}(n) \leq M n^{1+\epsilon}$. We show that there is a $\delta=\delta(\epsilon, g, K)>$ 0 such that $\operatorname{diam}(S)>\delta \sqrt{A(S)}$. This is where we use the bounds of Cheeger constants. To simplify let's say that $S$ is a sphere. By Proposition 2.3 there is a simple closed curve $p$ of length smaller than $4 \sqrt{A(S)}$ on $S$ which divides it into two pieces with comparable area. Now if $\operatorname{diam}(S)$ is small the filling area of $p$ is much smaller than $A(S)$. So one can subdivide $S$ into two 2-cycles of area roughly between $A(S) / 4$ and $3 A(S) / 4$. It follows by the convexity of $n^{1+\epsilon}$ that one of these two 2-cycles is also a counterexample to $F V_{3}(n) \leq M n^{1+\epsilon}$, a contradiction. Now given that the diameter of $S$ is big, we take a minimal volume 3-cycle $R$ filling $S$ and we fix a point $x$ on $S$. We consider 'balls' around $x$ in $R$, and again using the convexity of $n^{1+\epsilon}$ and an elementary inequality (Lemma 4.5) we see that the 2-cycle, say $S_{1}$, given by the boundary of some of these 'balls' has filling volume of the order of $A\left(S_{1}\right)^{3 / 2}$, contradicting the hypothesis of the theorem.

\section{Cheeger Constants of Surfaces}

If $M$ is a Riemannian manifold of dimension $n$, one defines the Cheeger constant $h$ of $M$ by

$$
h(M)=\inf _{A}\left\{\frac{\operatorname{vol}_{n-1}(\partial A)}{\operatorname{vol}_{n}(A)}: \operatorname{vol}_{n}(A) \leq \frac{1}{2} \operatorname{vol}_{n}(M)\right\},
$$

where $A$ ranges over all open subsets of $M$ with smooth boundary. If $M$ is a simplicial manifold one can define the Cheeger constant of $M$ similarly; now $A$ runs over all simplicial submanifolds of $M$. To be more precise, we take $A$ to be a union of closed $n$ simplices and we define $\partial A=A \cap \overline{M-A}$. In the simplicial setting we define $\operatorname{vol}_{n}(A)$ to be the number of $n$-simplices of $A$ and $\operatorname{vol}_{n-1}(\partial A)$ to be the number of $(n-1)$-simplices of $\partial A$.

We remark that this definition also makes sense if $M$ is more generally an $n$ dimensional simplicial complex. To make this definition coincide with the existing literature on graphs one should first take the barycentric subdivision and then 
calculate the Cheeger constant. However, as here we are only concerned with surfaces, we will not pass to barycentric subdivisions.

As usual we refer to $\mathrm{vol}_{2}$ as the area and $v^{\circ} l_{1}$ as the length. If $M$ is a simplicial 2-manifold or a 2-manifold with a Riemannian metric, we denote by $A(M)$ the area of $M$. Similarly if $p$ is a (simplicial or Riemannian) path, we denote by $l(p)$ the length of $p$.

As we mentioned in the Introduction it follows from work of Hersch 22 and Yang and Yau [39] (see also 24]) that there is a bound on the Cheeger constant of a closed surface that depends only on its area (see also [25] and [15] for similar results for graphs).

In this section we give an alternative approach to these results. We will first treat the simplicial case and then we will outline the argument in the Riemannian case. In both cases our proof is based on the Besicovitch lemma [2] (see also [20, sec. 4.28, p. 252; this lemma is sometimes referred to as Almgren's lemma, 8, [1]):

Lemma 2.1. Let $D$ be a Riemannian manifold homeomorphic to the disc and let $\gamma=\partial D$. Suppose $\gamma$ is split into 4 subpaths, $\gamma=\alpha_{1} \cup \alpha_{2} \cup \alpha_{3} \cup \alpha_{4}$. Let $d_{1}=d\left(\alpha_{1}, \alpha_{3}\right)$, $d_{2}=d\left(\alpha_{2}, \alpha_{4}\right)$. Then

$$
A(D) \geq d_{1} d_{2}
$$

We introduce some notation:

If $X$ is a simplicial complex and $K$ is a subcomplex of $X$, we denote by $\operatorname{star}(K)$ the subcomplex of $X$ consisting of all closed simplices intersecting $K$. We denote by $\operatorname{star}_{i}(K)$ the subcomplex obtained by repeating the star operation $i$ times. If $v$ is a vertex of $X$, we define the ball of radius $n$ and center $v, B_{v}(n)$, by $B_{v}(n)=\operatorname{star}_{n}(v)$.

We state below Besicovitch's lemma ([]) in the simplicial setting:

Lemma 2.2. Let $D$ be a simplicial disc and let $\gamma=\partial D$. Suppose $\gamma$ is split into 4 subpaths, $\gamma=\alpha_{1} \cup \alpha_{2} \cup \alpha_{3} \cup \alpha_{4}$. Let $d_{1}=d\left(\alpha_{1}, \alpha_{3}\right), d_{2}=d\left(\alpha_{2}, \alpha_{4}\right)$. Then

$$
A(D) \geq d_{1} d_{2} \text {. }
$$

Proof. We consider $\operatorname{star}\left(\alpha_{1}\right)$, and we remark that its boundary has at least $2 d_{2}$ edges. Since each closed 2-simplex in $\operatorname{star}\left(\alpha_{1}\right)$ intersects the boundary of $\operatorname{star}\left(\alpha_{1}\right)$ at at most 2 edges we conclude that $A\left(\operatorname{star}\left(\alpha_{1}\right)\right) \geq d_{2}$. Now we repeat $d_{1}$ times, i.e. we consider $\operatorname{star}_{i}\left(\alpha_{1}\right)$ for $i=1,2, \ldots, d_{1}$ and we remark as before that

$$
A\left(\operatorname{star}_{i}\left(\alpha_{1}\right)-\operatorname{star}_{i-1}\left(\alpha_{1}\right)\right) \geq d_{2} .
$$

It follows that $A(D) \geq d_{1} d_{2}$.

Remark 1 . The same inequality applies for disks with a cell complex structure in which all cells are polygons with 2 or 3 sides (bigons or triangles). Indeed the proof above applies in this case too.

We start with the inequality for the sphere where the idea of the proof is more transparent.

Proposition 2.3. Let $S$ be a Riemannian manifold or a simplicial complex homeomorphic to the 2-sphere. Then the Cheeger constant, $h(S)$, of $S$ satisfies the inequality

$$
h(S) \leq \frac{16}{\sqrt{A(S)}},
$$

where $A(S)$ is the area of $S$. 
Proof. We deal first with the simplicial case. We consider all simple closed curves $c$, on the 1-skeleton of $S$, such that $S-c=S_{1} \cup S_{2}$, and both $S_{1}, S_{2}$ have area larger than or equal to $\frac{A(S)}{4}$. We now show that this set is not empty. To show this we construct successively simplicial disks with larger and larger area and we show that eventually the boundary of such a disk has the required properties.

We start with a 2-simplex $\sigma$. If $A(S) \leq 4$ we can take for $c$ the boundary of $\sigma$. Otherwise we add to $\sigma$ another 2-simplex that has a common edge with $\sigma$, and so on. We remark that every time we add a 2 -simplex to a given simplicial disk $D$ either the complement is a disk or the complement has two connected components. In the case where the complement has two connected components we add to $D$ the component of the smallest area. In this way we again obtain a bigger disk. We continue this procedure until the area of the disk we obtain becomes bigger or equal to $A(S) / 4$. By construction the area of the disk will be smaller than $3 A(S) / 4$, so the boundary of this disk has the required properties.

Let $p$ be a curve, with the above property, which is of minimal length.

Let's say $S-p=S_{1} \sqcup S_{2}$ (where $S_{1}, S_{2}$ are open). Without loss of generality we assume that $A\left(S_{1}\right) \geq A\left(S_{2}\right)$.

We now remark that there are no 'shortcuts' for $p$ that are contained in $S_{1}$.

More precisely if $a, b \in p$ and $q$ is a path in $S_{1}$ joining $a, b$, then $l(q)$ is at least as big as the length of the shortest subpath of $p$ joining $a, b$. Indeed assume this is not the case. Let's say $p-\{a, b\}=p_{1} \cup p_{2}$. Without loss of generality we may assume that $q$ intersects $p$ only at $a, b$. Then $p_{1} \cup q$ or $p_{2} \cup q$ is a closed curve shorter than $p$ which has the same properties as $p$, a contradiction.

We claim that

$$
l(p) \leq 4 \sqrt{A(S)} .
$$

From Besicovitch's lemma $A\left(S_{1}\right), A\left(S_{2}\right) \geq l(p)-2$. So $A(S) \geq 2 l(p)-4$. It follows that the inequality

holds if $l(p) \leq 24$.

$$
l(p) \leq 4 \sqrt{A(S)}
$$

Otherwise we subdivide $p$ into $4 \operatorname{arcs} p=\alpha_{1} \cup \alpha_{2} \cup \alpha_{3} \cup \alpha_{4}$ such that

$$
l\left(\alpha_{i}\right) \geq \frac{l(p)-3}{4}
$$

for all $i$. Since there are no 'shortcuts' in $S_{1}$, as we observed above

$$
d\left(\alpha_{1}, \alpha_{3}\right) \geq \frac{l(p)-3}{4}, \quad d\left(\alpha_{2}, \alpha_{4}\right) \geq \frac{l(p)-3}{4} .
$$

Applying Lemma 2.2 to $S_{1}$ we have that

$$
\begin{aligned}
A\left(S_{1}\right) & \geq(l(p)-3)^{2} / 16 \Rightarrow 3 A(S) \geq(l(p)-3)^{2} / 4 \\
& \Rightarrow l(p) \leq \sqrt{12 A(S)}+3 \leq 4 \sqrt{A(S)},
\end{aligned}
$$

where the last inequality holds since $l(p) \geq 24$; hence $A(S) \geq 44$.

We conclude that

$$
h(S) \leq \frac{4 \sqrt{A(S)}}{A(S) / 4} \leq \frac{16}{\sqrt{A(S)}} .
$$

We now treat the Riemannian case. The argument is along the same lines. To sidestep the issue of existence and regularity of the minimal closed curve $p$ we argue instead with $\epsilon$-minimal curves. More precisely we consider the set $U$ of all simple closed curves dividing $S$ in two discs in which both have area larger than or equal 
to $\frac{A(S)}{4}$. Let $L$ be the infimum of the lengths of the curves in $U$. Given $\epsilon>0$ we consider $p \in U$ with $l(p)<L+\epsilon$. Let's say that $S-p=S_{1} \sqcup S_{2}$ and $A\left(S_{1}\right) \geq A\left(S_{2}\right)$. Then $p$ does not have $\epsilon$-shortcuts in $S_{1}$. That is, if $q \subset S_{1}$ is a path joining $a, b \in p$, then $l(q)-\epsilon$ is not smaller than the length of the shortest subpath of $p$ joining $a, b$.

We now subdivide $p$ in $4 \operatorname{arcs} p=\alpha_{1} \cup \alpha_{2} \cup \alpha_{3} \cup \alpha_{4}$ such that $l\left(\alpha_{i}\right)=l(p) / 4$ for all $i$. Since there are no $\epsilon$-'shortcuts' as we observed above,

$$
d\left(\alpha_{1}, \alpha_{3}\right) \geq \frac{l(p)}{4}-\epsilon, \quad d\left(\alpha_{2}, \alpha_{4}\right) \geq \frac{l(p)}{4}-\epsilon
$$

Applying Lemma 2.1 to $S_{1}$ we have that

$$
A\left(S_{1}\right) \geq(l(p)-4 \epsilon)^{2} / 16 \Rightarrow 12 A(S) \geq(l(p)-4 \epsilon)^{2} \Rightarrow l(p) \leq 2 \sqrt{3 A(S)}+4 \epsilon .
$$

It follows that

$$
h(S) \leq \frac{2 \sqrt{3 A(S)}+4 \epsilon}{A(S) / 4} \Rightarrow h(S) \leq \frac{8 \sqrt{3}}{\sqrt{A(S)}},
$$

where the last inequality follows since the former inequality holds for every $\epsilon>0$. We note that we obtain a better constant in the Riemannian case.

Remark 2. The same inequality for the Cheeger constant applies to spheres with a cell complex structure in which all cells are polygons with 2 or 3 sides (bigons or triangles). Indeed the proof above applies in this case too.

To treat the general case of compact surfaces we need some technical lemmas.

Definition. Let $S$ be a compact surface with boundary. A simple arc $p$ intersecting the boundary only at its endpoints is said to be parallel to the boundary if $S-p$ has a contractible component. Two disjoint simple arcs $p_{1}, p_{2}$ intersecting the boundary only at their endpoints are said to be parallel if $S-\left(p_{1} \cup p_{2}\right)$ has a contractible component.

Lemma 2.4. Let $S$ be a surface of genus $g$ with $k$ boundary components. Then there are at most $6 g+3 k$ pairwise disjoint arcs on $S$ with their endpoints on $\partial S$ such that no arc is parallel to the boundary and no two arcs are parallel.

Proof. To show this lemma we will show something slightly more general. We say that $\Sigma$ is a marked surface with $m$ arcs, if $\Sigma$ is a connected surface with boundary, equipped with disjoint $\operatorname{arcs} \alpha_{1}, \ldots, \alpha_{m}$, which are contained in the boundary of $\Sigma$.

Now let $\beta$ be a simple arc on $\Sigma$ intersecting the boundary only at its endpoints. We also assume that $\beta$ does not intersect any of the $\alpha_{i}$ 's.

We say that $\beta$ is parallel to the marked boundary if $\Sigma-\beta$ has a component that is a disk and which contains at most one of the $\alpha_{i}$ 's. We say that two disjoint arcs $\beta_{1}, \beta_{2}$ are parallel in $\Sigma$ if a component of $\Sigma-\left(\beta_{1} \cup \beta_{2}\right)$ is a disc whose boundary contains $\beta_{1}, \beta_{2}$ and which does not intersect any $\alpha_{i}$.

Now let $\Sigma$ be a marked surface of genus $g$, with $k$ boundary components and $m>0$ arcs. We claim that there are at most $6 g+3 k+m-3$ pairwise disjoint arcs with endpoints on $\partial \Sigma$ such that no arc is parallel to the marked boundary and no two arcs are parallel in $\Sigma$. We will prove this by induction on $6 g+3 k+m-3$. If $6 g+3 k+m-3=0$, then either $m=3$ or $k=1, m=0$. In both cases there are no arcs non-parallel to the boundary.

Now let $U$ be a collection of arcs in $\Sigma$ which are not parallel to the marked boundary and are not pairwise parallel. We cut $\Sigma$ along an $\operatorname{arc} \beta$ in $U$, and we obtain a surface $\Sigma^{\prime}$. We distinguish 3 cases. 
1 st case. The genus decreases by 1 to $g-1$. Then $k$ increases to $k+1$ and $m$ increases to $m+2$. We have

$$
6(g-1)+3(k+1)+m+2=6 g+3 k+m-1 .
$$

Since $U-\{\beta\}$ is a collection of non-parallel and non-boundary parallel arcs in $\Sigma^{\prime}$, the assertion follows by induction.

$2 n d$ case. $\beta$ is separating, so $\Sigma^{\prime}$ has two connected components $\Sigma_{1}, \Sigma_{2}$, which are marked surfaces. We denote by $g_{1}, g_{2}, k_{1}, k_{2}, m_{1}, m_{2}$ respectively, the genus, number of boundary components and marked arcs of $\Sigma_{1}, \Sigma_{2}$. We then have

$$
g_{1}+g_{2}=g, k_{1}+k_{2}=k, m_{1}+m_{2}=m+2 .
$$

So

$$
6 g_{1}+3 k_{1}+m_{1}-3+6 g_{2}+3 k_{2}+m_{2}-3=6 g+3 k+m-4,
$$

and the assertion follows again by induction.

$3 r d$ case. $\beta$ joins two distinct boundary components. In this case $k$ decreases to $k-1$ and $m$ increases to $m+2$. We have for $\Sigma^{\prime}$

$$
6 g+3(k-1)+m+2=6 g+3 k+m-1,
$$

so the assertion follows again by induction.

We return now to the proof of the lemma. If we cut $S$ along an arc we obtain a marked surface $\Sigma^{\prime}$ with 2 arcs. If we denote by $g^{\prime}, k^{\prime}$ the genus and number of boundary components of $\Sigma$, we have

$$
6 g^{\prime}+3 k^{\prime}+2 \leq 6 g+3 k-1
$$

examining cases as before. We now remark that a set of non-parallel and nonboundary parallel arcs in $S$ gives a set of non-parallel and non-boundary parallel $\operatorname{arcs}$ in $\Sigma$ after we cut $S$ by one of these arcs. So the lemma follows.

Lemma 2.5. Let $S$ be a closed orientable surface of genus $g$. If $U=\left\{p_{1}, \ldots, p_{k}\right\}$ is a set of pairwise disjoint simple closed curves on $S$, such that $S-U$ has exactly 2 connected components, then $k \leq g+1$.

Proof. Since $S-U$ has exactly 2 connected components, at most one of the $p_{i}$ 's is separating. Cutting $S$ by any other $p_{i}$ reduces genus by 1 . Since the genus of $S$ is $g$, we have that $k \leq g+1$.

Proposition 2.6. Let $S$ be a closed orientable surface of genus $g \geq 1$ equipped either with a Riemannian metric or with a simplicial complex structure. Let $A(S)$ be its (simplicial or Riemannian) area. Then the Cheeger constant, $h(S)$, of $S$ satisfies the inequality

$$
h(S) \leq \frac{4 \cdot 10^{3} \cdot g^{2}}{\sqrt{A(S)}}
$$

Proof. We first treat the simplicial case. The proof in the Riemannian case follows the same lines; we outline at the end the changes which are needed in this case.

Let $U=\left\{p_{1}, \ldots, p_{k}\right\}$ be a set of closed curves on the 1-skeleton of $S$ such that:

1. $S=S_{1} \cup S_{2}$ with $\partial S_{1} \cap \partial S_{2}=S_{1} \cap S_{2}=U, A\left(S_{i}\right) \geq A(S) / 4, i=1,2$.

2. The interiors of $S_{1}, S_{2}$ are connected surfaces.

3. The sum of the lengths $L=l\left(p_{1}\right)+\ldots+l\left(p_{k}\right)$ is minimal among all sets of curves satisfying 1,2 . 
We now show that such a set of curves exists. This is similar to the argument in Proposition 2.3 .

We start with a 2 -simplex, and we successively add adjacent 2 -simplices. If we denote by $\Sigma$ the subset of $S$ obtained at a given step, we have that the interior of $\Sigma$ is an open surface while the boundary of $\Sigma$ is a union of closed curves. We note that these curves are not necessarily simple, but if we push them to the interior of $\Sigma$ they become simple. As we noted in Proposition 2.3 when we add a simplex to $\Sigma$ its complement might become disconnected. In this case we add the component of smallest area to $\Sigma$ (if both components have equal area we add any one of the two). We continue this procedure until the area of $\Sigma$ becomes larger than or equal to $A(S) / 4$. By construction its area will also be smaller than $3 A(S) / 4$. Since, by construction, the complement of $\Sigma$ is connected, it follows from Lemma 2.5 that the boundary of $\Sigma$ can be written as a union of at most $g+1$ closed curves. It follows that

We claim that

$$
k \leq g+1
$$

$$
L \leq 10^{3} \cdot g^{2} \cdot \sqrt{A(S)}
$$

Suppose that this is not the case. Then there is a curve $p_{i} \in U$ such that

$$
l\left(p_{i}\right) \geq \frac{10^{3} g^{2} \sqrt{A(S)}}{g+1} .
$$

We set $n=[\sqrt{A(S)}]+1$. Let's assume that $A\left(S_{1}\right) \geq A\left(S_{2}\right)$.

We now pick a vertex $v \in p_{i}$. We claim that $S_{1}$ is not contained in the ball of radius $3 n$ and center $v, B_{v}(3 n)$. Suppose not. We remark that $S_{1}$ has genus bounded by $g$ and has at most $g+1$ boundary components by Lemma 2.5.

We subdivide $p_{i}$ at $6 g+3(g+1)+1=9 g+4$ segments of length larger than

$$
\left[\frac{10^{3} g^{2} \sqrt{A(S)}}{(g+1)(9 g+4)}\right] \geq 10 n
$$

We consider geodesic arcs in $S_{1}$ from $v$ to the endpoints of these segments. If some such arc is parallel to the boundary of $S_{1}, \partial S_{1}$, we can use it to 'cut away' a disc from $S_{1}$ and contradict the minimality of $U$ (or in case the disc has area more than half of the area of $S_{1}$, we replace $S_{1}$ by the disc and contradict property 3 ). Similarly we arrive at a contradiction if some arc is separating $S_{1}$; we can again cut away a piece of $S_{1}$ with smaller boundary length.

Otherwise by perturbing these arcs slightly we may arrange it so that they are disjoint. Since we have $9 g+4 \operatorname{arcs}$ by Lemma 2.4. two of them are parallel. Using them we can cut away a disk from $S_{1}$ (or replace $S_{1}$ by a disc) which contradicts the minimality of $U$ (property 3 ). We remark that since no arc is separating the new regions we obtain after this operation satisfy $1,2,3$.

We now consider $D_{r}=B_{v}(r) \cap S_{1}$ for $n<r \leq 2 n$. We remark that if the length of $\partial D_{r}-\partial S_{1}$ is larger than $2 n$ for all $r$, then $A\left(D_{n}\right) \geq n^{2} \geq A(S)$, a contradiction.

So $\partial D_{r}-\partial S_{1}$ has length less than $2 n$ for some $r, n<r \leq 2 n$. On the other hand the length of $D_{r} \cap p_{i}$ is larger than $2 n$. So $S_{1}-D_{r}$ and $D_{r}$ both have boundary length smaller than the length of $\partial S_{1}$. Let $w$ be a vertex of $p_{i}$ that does not lie in $B_{v}(3 n)$ and let $C$ be the component of $S_{1}-D_{r}$ that contains $w$. Clearly either $C$ or $S_{1}-C$ has area larger than or equal to $A\left(S_{1}\right) / 2 \geq A(S) / 4$. Moreover the boundary of each one of them has smaller length than the boundary of $S_{1}$, and the 
complement of each one of them is connected. This contradicts the minimality of $U$ (property 3 ).

The same proof applies in the Riemannian case with few changes. We define a set of closed curves $U=\left\{p_{1}, \ldots, p_{k}\right\}$ as before. Now we may additionally assume that the $p_{i}$ are simple and disjoint. To insure this and avoid existence issues, we assume that the sum of their lengths exceeds the minimal possible value by at most $\epsilon>0$ (condition 3 ) among all curves that satisfy 1,2. As before we argue that there is some $p_{i}$ such that

$$
l\left(p_{i}\right) \geq \frac{10^{3} g^{2} \sqrt{A(S)}}{g+1}-\epsilon .
$$

We argue as before, and we consider $D_{r}=B_{v}(t) \cap S_{1}$ for $r \in[n, 2 n]$. Now by the co-area formula if the length of $\partial D_{r}-\partial S_{1}$ is larger than $2 n$ for almost all $r$, then $A\left(D_{n}\right) \geq n^{2} \geq A(S)$, which gives a contradiction as before. The rest of the proof applies verbatim to the Riemannian case as well.

Remark 3. We remark that there is no function of volume that gives an upper bound for the Cheeger constant of manifolds of dimension higher than 2. Indeed it's enough to prove this for the ball of dimension 3. We can obtain examples contradicting the existence of such a bound by considering sequences of expanders and thickening them.

\section{ISOPERIMETRIC PROFILES OF SURFACES}

If $\left(M^{n}, g\right)$ is a Riemannian manifold of infinite volume, the isoperimetric profile function of $M^{n}$ is a function $I_{M}: \mathbb{R}^{+} \rightarrow \mathbb{R}^{+}$defined by

$$
I_{M}(t)=\inf _{\Omega}\left\{\operatorname{vol}_{n-1}(\partial \Omega): \Omega \subset M^{n}, \operatorname{vol}_{n}(\Omega)=t\right\},
$$

where $\Omega$ ranges over all regions of $M^{n}$ with smooth boundary. One can similarly define an isoperimetric profile function $I_{M}: \mathbb{N} \rightarrow \mathbb{N}$ for simplicial manifolds $M^{n}$. In this section we will study isoperimetric profiles and filling functions of surfaces (so $\mathrm{vol}_{2}$ is area and $v l_{1}$ is length).

Other functions related to the isoperimetric problem are the filling area, $F A_{0}$, and homological filling area, $F A^{h}$, functions of $M$ that we define now.

If $p$ is a smooth contractible closed curve in $M$, we define its filling area, Fill $_{0}(p)$, as follows: We consider all Riemannian discs $D$ such that there is a 1-Lipschitz map $f: D \rightarrow X$ with $\left.f\right|_{\partial D}=p$. We define $F_{i l l} A_{0}(p)$ to be the infimum of the areas of this collection of disks. We now define the filling area function of $M$ by

$$
F A_{0}(t)=\sup _{p}\left\{F_{i l l} A_{0}(p): l(p) \leq t\right\}
$$

where $p$ ranges over all smooth contractible closed curves of $M$.

More generally we can consider 1-cycles $c$ (i.e. unions of closed curves) that can be filled by 2 -cycles to define the homological filling area function. To define Fill $A(c)$ we consider surfaces with boundary $(S, \partial S)$, equipped with a Riemannian metric, such that there is a 1-Lipschitz map $f: S \rightarrow X$ with $\left.f\right|_{\partial S}=c$. We then define

$$
F A^{h}(t)=\sup _{c}\{F i l l A(c): l(c) \leq t\}
$$


where if $c=c_{1} \sqcup \ldots \sqcup c_{n}$ with $c_{i}$ closed curves, we define $l(c)=l\left(c_{1}\right)+\ldots+l\left(c_{n}\right)$. One defines $F A_{0}$ and $F A^{h}$ similarly in the simplicial setting as well.

Gromov (18, ch. 6; see also [10, ch.6) showed the following:

Gromov's theorem. Let $\left(M^{n}, g\right)$ be a simply connected Riemannian manifold. Assume that there is some $t_{0}$ such that for all $t>t_{0}, F A_{0}(t) \leq \frac{1}{16 \pi} t^{2}$. Then there is a constant $K$ such that for all $t>t_{0}, F A_{0}(t) \leq K t$.

We remark that Wenger ([37]) improved $\frac{1}{16 \pi}$ to $\frac{1-\epsilon}{4 \pi}$ (for any $\epsilon>0$ ), which is optimal as the example of the Euclidean plane shows. In fact Gromov's theorem applies more generally to 'reasonable' geodesic metric spaces where a notion of area can be defined (e.g. simplicial complexes). We note also that Gromov has shown a stronger ('effective') version than the one we state; it is enough in fact to have a subquadratic filling for a sufficiently large range of areas to conclude that the filling is linear.

In the case of surfaces the isoperimetric profile and the filling area functions are closely related. In fact $F A_{0}$ is linear for a space if and only if the space is Gromov hyperbolic (see [18]). On the other hand if a simply connected surface $S$, equipped with a Riemannian metric, is not Gromov hyperbolic, then for any $t$ there is an embedded loop $\gamma$ in $S$ with $l(\gamma)>t$ such that Fill $A_{0}(\gamma)>\frac{1}{16 \pi} l(\gamma)^{2}$. If $t_{1}=$ Fill $_{0}(\gamma)$ we see that for any $t>0$ there is some $t_{1}>t$ such that $I_{S}\left(t_{1}\right)<$ $4 \sqrt{\pi} \sqrt{t}_{1}$.

Theorem 3.1. Let $(S, g)$ be a simply connected Riemannian surface. Assume that there is some $t_{0}$ such that for all $t>t_{0}, I_{S}(t) \geq 4 \sqrt{\pi} \sqrt{t}$. Then there is a constant $\delta>0$ such that for all $t>t_{0}, I_{S}(t) \geq \delta t$.

We remark that in many cases $F A_{0}$ and $F A^{h}$ are equal (e.g. this holds for the Euclidean and hyperbolic planes).

This does not always hold however. We now give some examples to illustrate the relationship between the filling area functions and the isoperimetric profile. If $f(t), g(t)$ are functions we write $f(t) \sim g(t)$ if

$$
\limsup _{t \rightarrow \infty} \frac{f(t)}{g(t)}<\infty, \quad \liminf _{t \rightarrow \infty} \frac{f(t)}{g(t)}>0 .
$$

Example 3.2. Let $X$ be the punctured Euclidean plane $\mathbb{R}^{2}-\mathbb{Z}^{2}$. Then $F A_{0}(t) \sim t$ (see [3], [31]), while $F A^{h}(t) \sim t^{2}$. The isoperimetric profile $I_{X}$ is the inverse of $F A^{h}$, so $I_{X}(t) \sim \sqrt{t}$.

Example 3.3. Let $X$ be the cylinder $S^{1} \times \mathbb{R}$ with the standard product metric. Then $F A_{0}(t) \sim t^{2}$. Indeed for any $X, F A_{0}$ is the same for $X$ and for the universal covering $\tilde{X}$. Here $\tilde{X}=\mathbb{E}^{2}$ (the Euclidean plane). On the other hand if $s$ is the length of the $\mathbb{S}^{1}$ factor for any $t>2 s$, we have $F A^{h}(t)=\infty$. Similarly for the isoperimetric profile there is some $t_{0}$ such that for all $t>t_{0}, I_{X}(t)=2 s$.

Example 3.4. Let $X_{n}$ be the space obtained by removing a ball of radius $n$ from the hyperbolic plane $\mathbb{H}^{2}$. Let $X$ be isometric to the hyperbolic plane. We fix a point $O \in X$ and we consider a sequence of points $x_{n}$ such that $d\left(x_{n}, O\right)=2^{n}$. For each $n$ we remove the disk of radius $n$ and center $x_{n}$ from $X$, and we glue along the boundary of the disk a copy of $X_{n}$. The space $Y$ obtained has $F A_{0}(t) \sim F A^{h}(t) \sim$ $I_{Y}(t) \sim t$. We remark that $Y$ is not Gromov hyperbolic. 
It is reasonable to ask whether Theorem 3.1 extends to other surfaces. We show below that the theorem holds for surfaces of bounded genus (this applies for example to Riemannian planes with infinitely many holes; compare 31]). We have the following:

Theorem 3.5. Let $S$ be a plane with holes equipped either with a Riemannian metric or with a simplicial complex structure. Assume that there is some $K>0$ such that for all $t \in[K, 100 K], I_{S}(t) \geq 10^{2} \sqrt{t}$. Then for all $t>K, I_{S}(t) \geq \frac{1}{\sqrt{K}} t$.

Proof. We treat the simplicial case first.

We argue by contradiction. We consider the set of all embedded 1-cycles $a$ with Fill $A(a)>K$ such that $l(a)<\frac{1}{\sqrt{K}}$ Fill $A(a)$. Let $c$ be an element of this set with minimal filling area.

Let's say that $c=\partial R$. We claim that Fill $A(c)>100 K$. Indeed if Fill $A(c) \leq$ $100 K$, then $l(c) \geq 100 \sqrt{\text { Fill } A(c)}$; hence

$$
\frac{1}{\sqrt{K}} \text { Fill } A(c)>100 \sqrt{\text { FillA(c) }} \Rightarrow \text { Fill A }(c)>10^{4} K .
$$

By our minimality assumption $R$ is connected, so $R$ is a sphere with holes (possibly a disc). We collapse all holes to points, and we obtain a sphere $\Sigma$ with a cell complex structure in which all cells are either bigons or triangles.

In this way we obtain a map $f: R \rightarrow \Sigma$ which is $1-1$ on open 2 -simplices from $R$.

By Proposition 2.3 (and the remark following it) there is a simple closed curve $p$ in $\Sigma^{1}$ such that Fill $A(p) \geq A(\Sigma) / 4$ and

$$
l(p) \leq 4 \sqrt{A(\Sigma)} .
$$

It follows that

$$
\operatorname{Fill} A(p) \geq \frac{1}{64} l(p)^{2} .
$$

We now consider the set of curves $q$ in $\Sigma^{1}$ with filling area $\operatorname{Fill} A(q) \geq K$ that satisfy Fill $A(q)>\frac{1}{100} l(q)^{2}$. Clearly this set is not empty. Let $\gamma$ in $\Sigma^{1}$ be of minimal filling area with this property. We will show that $4 K \geq \operatorname{Fill} A(\gamma) \geq K$. Assume this is not the case. $i$.

We subdivide $\gamma$ in $4 \operatorname{arcs} \gamma=\alpha_{1} \cup \alpha_{2} \cup \alpha_{3} \cup \alpha_{4}$ such that $l\left(\alpha_{i}\right) \geq \frac{l(\gamma)}{4}-1$ for all

We claim that

$$
d\left(\alpha_{1}, \alpha_{3}\right) \geq \frac{l(\gamma)}{16}, \quad d\left(\alpha_{2}, \alpha_{4}\right) \geq \frac{l(\gamma)}{16} .
$$

We argue by contradiction. Assume that $d\left(\alpha_{1}, \alpha_{3}\right)<\frac{l(\gamma)}{16}$ and let $w$ be a path from $\alpha_{1}$ to $\alpha_{3}$ of length $r<\frac{l(\gamma)}{16}$. Then, using $w$, we split $\gamma$ into two curves $\gamma_{1}, \gamma_{2}$ such that $\gamma_{1} \cap \gamma_{2}=w, \gamma_{1} \cup \gamma_{2}=\gamma \cup w$. Let's say $l\left(\gamma_{1}\right) \geq l\left(\gamma_{2}\right)$. Then

$$
l\left(\gamma_{1}\right)=\frac{l(\gamma)}{2}+a+r, \quad l\left(\gamma_{2}\right)=\frac{l(\gamma)}{2}-a+r
$$

for some $a \leq l(\gamma) / 4$. To simplify the notation we set $l=l(\gamma)$. We claim that Fill $A\left(\gamma_{1}\right)>K$, Fill $A\left(\gamma_{2}\right)>K$. Indeed by our assumption Fill $A(\gamma)>4 K$. If, say, $\operatorname{Fill} A\left(\gamma_{2}\right) \leq K$, then

$$
\operatorname{Fill} A\left(\gamma_{1}\right) \geq \frac{3 \text { FillA }(\gamma)}{4} \geq \frac{3 l^{2}}{400}>\frac{1}{100} l\left(\gamma_{1}\right)^{2},
$$


and this contradicts our minimality assumption on $\gamma$. Similarly we arrive at a contradiction if Fill $A\left(\gamma_{1}\right) \leq K$ since $l\left(\gamma_{1}\right) \geq l\left(\gamma_{2}\right)$. We now have

$$
\begin{gathered}
\text { FillA }(\gamma) \leq \text { FillA }\left(\gamma_{1}\right)+\text { Fill } A\left(\gamma_{2}\right) \leq \frac{1}{100}\left[\left(\frac{l}{2}+a+r\right)^{2}+\left(\frac{l}{2}-a+r\right)^{2}\right] \\
=\frac{1}{100}\left(\frac{l^{2}}{2}+2 a^{2}+2 r^{2}+2 l r\right) .
\end{gathered}
$$

Since $a \leq \frac{l}{4}, r \leq \frac{l}{16}$ we have

$$
\frac{l^{2}}{2}+2 a^{2}+2 r^{2}+2 l r \leq \frac{l^{2}}{2}+\frac{l^{2}}{8}+\frac{2 l^{2}}{16^{2}}+\frac{l^{2}}{8}<l^{2},
$$

which contradicts our assumption that

$$
\operatorname{Fill} A(\gamma)>\frac{1}{100} l^{2}
$$

We may now apply Lemma 2.2 to $\gamma$ and conclude that

$$
\text { Fill } A(\gamma) \geq\left(\frac{l}{4}-1\right) \frac{l}{16},
$$

which contradicts our assumption that $\operatorname{Fill} A(\gamma)$ is minimal with the property

$$
\operatorname{Fill} A(\gamma)>\frac{1}{100} l^{2}
$$

We conclude that $4 K \geq \operatorname{Fill} A(\gamma) \geq K$.

We now lift $\gamma$ via $f$ to $R$. $\gamma$ lifts to a set of arcs (or a single simple closed curve) that separate $R$ into two 2-chains $R_{1}, R_{2}$. Let's denote this set of arcs by $\alpha$. Let's say that $A\left(R_{2}\right)>A\left(R_{1}\right)$, so $K \leq A\left(R_{1}\right) \leq 4 K$. Then $\partial R_{1}=c_{1} \cup \alpha$ and $\partial R_{2}=c_{2} \cup \alpha$ with $c_{1} \cup c_{2}=c$. By our assumption on $c$ we have

$$
l\left(c_{1}\right)+l\left(c_{2}\right) \leq \frac{1}{\sqrt{K}}\left(A\left(R_{1}\right)+A\left(R_{2}\right)\right) .
$$

On the other hand, since $c$ is minimal with this property we have

$$
\begin{aligned}
& l\left(c_{1}\right)+l(\alpha) \geq \frac{1}{\sqrt{K}} A\left(R_{1}\right), \\
& l\left(c_{2}\right)+l(\alpha) \geq \frac{1}{\sqrt{K}} A\left(R_{2}\right) .
\end{aligned}
$$

By the way $\alpha$ was defined we have

$$
A\left(R_{1}\right) \geq \frac{1}{100} l(\alpha)^{2} .
$$

Since $A\left(R_{1}\right) \leq 4 K$ we have $l(\alpha) \leq 20 \sqrt{K}$. From the hypothesis of the theorem, since $A\left(R_{1}\right) \in[K, 100 K]$ we have

$$
100 \sqrt{A\left(R_{1}\right)} \leq l(\alpha)+l\left(c_{1}\right) \Rightarrow l\left(c_{1}\right) \geq 80 \sqrt{K} .
$$

Substituting in (1) we obtain

$$
l\left(c_{2}\right) \leq \frac{1}{\sqrt{K}}\left(4 K+A\left(R_{2}\right)\right)-80 \sqrt{K} .
$$

Therefore

$$
l\left(c_{2}\right) \leq \frac{1}{\sqrt{K}} A\left(R_{2}\right)-76 \sqrt{K},
$$


and from (3)

$$
\frac{1}{\sqrt{K}} A\left(R_{2}\right)-76 \sqrt{K}+20 \sqrt{K} \geq \frac{1}{\sqrt{K}} A\left(R_{2}\right),
$$

which is a contradiction.

The proof in the Riemannian case is identical. One has just to note that when we collapse the boundary curves to points we obtain a Riemann metric with some singularities. The estimates for Cheeger constants apply however to this case as well. One can see this e.g. by approximating the singular metric by a non-singular one or by noting that the proof we gave of the Cheeger constant bound also work for singular metrics.

Corollary 3.6. Let $S$ be a complete non-compact surface of finite genus equipped either with a Riemannian metric or with a simplicial complex structure. Assume that there is some $K>0$ such that for all $t \in[K, 100 K], I_{S}(t) \geq 10^{2} \sqrt{t}$. Then there is a constant $\delta>0$ such that for all $t>K, I_{S}(t) \geq \delta t$.

Proof. There is a finite set $\left\{p_{1}, \ldots, p_{n}\right\}$ of smooth, rectifiable, simple closed curves (or a finite set of simple closed curves lying in the 1-skeleton, $S^{(1)}$, in the simplicial case) such that

$$
S-\left\{p_{1}, \ldots, p_{n}\right\}=B \sqcup B_{1} \sqcup \ldots \sqcup B_{k}
$$

with $B$ a surface of finite area and $B_{1}, \ldots, B_{k}$ planes with holes. From the previous theorem we have that there are $\delta_{1}, \ldots, \delta_{k}$ such that for all $t>K$ we have

$$
I_{B_{i}}(t) \geq \delta_{i} t, \quad i=1, \ldots, k \text {. }
$$

Let $\delta^{\prime}=\min \left\{\delta_{1}, \ldots, \delta_{k}\right\}$. We set $V=A(B)$ and $L=l\left(p_{1}\right)+\ldots+l\left(p_{n}\right)$.

If $\Omega$ is a domain in $S$ with rectifiable boundary, there is some $i \in\{1, \ldots, k\}$ such that

$$
A\left(\Omega \cap B_{i}\right) \geq \frac{A(\Omega)-V}{k} .
$$

Let $c=\partial \Omega \cap B_{i}$. Since $\partial\left(\Omega \cap B_{i}\right) \subset c \cup\left\{p_{1}, \ldots, p_{n}\right\}$ we have that $l\left(\partial\left(\Omega \cap B_{i}\right)\right) \leq$ $L+l(c)$. If

$$
\frac{A(\Omega)-V}{k}>K \Leftrightarrow A(\Omega)>k K+V
$$

we have

$$
L+l(c) \geq \delta^{\prime} \frac{A(\Omega)-V}{k} \Rightarrow l(c) \geq \delta^{\prime} \frac{A(\Omega)-V}{k}-L .
$$

It follows that if

$$
\delta^{\prime} \frac{A(\Omega)}{k} \geq 2\left(\frac{\delta^{\prime} V}{k}+L\right) \Leftrightarrow A(\Omega) \geq 2 V+\frac{2 k L}{\delta^{\prime}}
$$

we have

$$
l(\partial \Omega) \geq l(c) \geq \delta^{\prime} \frac{A(\Omega)}{2 k} .
$$

We conclude that for all

$$
t>K_{1}=\max \left(k K+V, 2 V+\frac{2 k L}{\delta^{\prime}}\right)
$$

we have

$$
I_{S}(t) \geq \frac{\delta^{\prime}}{2 k} t
$$


We note further that if $\delta_{1}=\inf \left\{I_{S}(t): t>K\right\}$ and

$$
\delta=\min \left(\frac{\delta_{1}}{K_{1}}, \frac{\delta^{\prime}}{2 k}\right)
$$

then for all $t>K$ we have

$$
I_{S}(t) \geq \delta t
$$

Remark 4. The previous 'gap' theorem does not generalize to all surfaces. We now sketch a way to see this. Consider the Euclidean disc and drill (equal) holes to it so that the area decreases. Clearly if the radius of the disc is $R$ one may drill holes so that the area becomes $R^{a}$ for any $a>1$. Now one glues the holes 'randomly' (this is similar to the construction of expander graphs). In this way one obtains a surface with boundary with isoperimetric profile which satisfies $I(R)=R^{1 / 2+\epsilon}$ for any $\epsilon>0$ by varying $a$. Now take a hyperbolic plane and a sequence of disks with radii that tend to infinity, cut out the disks and glue in the surfaces we constructed above. This gives examples of surfaces with 'intermediate' isoperimetric profile function.

Remark 5. The previous theorem implies that if the filling area function is subquadratic for surfaces of finite genus, then it is actually linear. In fact one may give a similar proof to another generalization of Gromov's theorem. Let $X$ be either a Riemannian manifold or a simplicial complex. Let $c$ be a 1-cycle. If $c=\partial R$ for some 2-chain $R$, then we define the genus of $R$ to be the genus of the 2-cycle we obtain from $R$ by collapsing $c$ to a point. If $c$ is a 1-cycle in $X$ we define the $g$-filling area of $c$ by

$$
\text { Fill } A_{g}(c)=\inf \{A(S): S \text { is a 2-chain of genus at most } g \text { s.t. } \partial S=c\} \text {. }
$$

Note that with this definition Fill $A_{0}$ is slightly more general than before, as it applies to 1-cycles and not just closed curves. However, this does not affect what follows. We now define the $g$-filling area of $X$ by

$$
F A_{g}(t)=\sup _{c}\left\{F i l l A_{g}(c): c=\partial R, R \text { of genus } \leq g \text { and } l(c) \leq t\right\} .
$$

With this notation Gromov's theorem says that if $F A_{0}$ is subquadratic, then it is bounded by a linear function. In fact now we can generalize this for any $g$ : For any $g$ there is some $\epsilon_{g}>0$ such that if for some $t_{0}, F A_{g}(t) \leq \epsilon_{g} t$ for all $t>t_{0}$, then there is some $K>0$ such that $F A_{g}(t) \leq K t$ for all $t>t_{0}$. The proof goes along the same lines as the proof of Theorem 3.5. We argue by contradiction assuming that we have a minimal 1-cycle $c$ that violates the linear isoperimetric inequality. We fill it by a minimal area 2 -chain $S$ of genus at most $g$. Then we collapse all boundary components of $S$ to obtain a closed 2-cycle $\Sigma$ of genus $\leq g$. Using Proposition 2.6 we show that we can 'cut' a 2-chain from $\Sigma$ with small boundary length and big area. We lift this back to $S$, and we argue as in Theorem 3.5 to contradict our assumption that $c$ is minimal.

We note finally that if one considers the function $F A^{h}$ rather than $F A_{g}$ (i.e. if one does not impose any bound on $g$ ), then there is no such gap between subquadratic and linear. 


\section{ISOPERIMETRIC INEQUALITIES}

In this section we will study the question of whether Gromov's 'gap' theorem for $F A_{0}$ extends to the 3 dimensional filling function $F V_{3}$. The filling area function $F A_{0}$ is important for group theory since it is related to the word problem. In fact if $G$ is a finitely presented group and $X$ is its Cayley complex, then $G$ has a solvable word problem if and only if $F A_{0}(X)$ is bounded by a recursive function (see 13 , thm. 2.2.5). We note that $X$ might not be a simplicial complex, but one can pass to a simplicial subdivision to make sense of $F A_{0}(X)$. The question of whether there are other 'gaps' for $F A_{0}$ apart from between $n$ and $n^{2}$ for finitely presented groups was answered in the negative (see [34, 6], 4]). It is easy to see that one can produce simplicial (or Riemannian) planes with $F A_{0}$ of the form, say, $n^{r}, r \in(2, \infty)$. For example one might start with the Euclidean plane, cut off a sequence of disjoint discs with larger and larder radii and glue back cylindrical caps ('mountains'). Grimaldi and Pansu ([16] study the finer question of characterizing completely filling functions for Riemannian planes.

Gromov (19]) has given estimates and formulated conjectures for higher dimensional filling functions of nilpotent groups (see also 36] and [5] for interesting examples of higher filling functions of groups).

We note that as we move to higher dimensions we have two possible ways to define filling functions. We can either define them by considering fillings of (singular) spheres by balls or more generally one may consider filling of higher dimensional cycles (e.g. filling of orientable surfaces of genus $g \geq 0$ in dimension 3 ). Here we take the second option; apart from being easier to define technically it seems more natural. For example, as it is shown in 30] that filling of 2-spheres in groups is always subrecursive, in contrast to $F A_{0}$, which is not subrecursive for groups with an unsolvable word problem. So examining filling only of 2 -spheres seems quite restrictive.

We refer to the Introduction for the definition of the terms in the theorem below. To simplify notation we denote Fillvol $_{2}(c)$ by FillA(c) if $c$ is a 1-cycle and Fillvol $_{3}(S)$ by FillV $(S)$ if $S$ is a 2-cycle.

Theorem 4.1. Let $X$ be a simplicial complex such that $H_{1}(X)=H_{2}(X)=0$. Assume that the following hold:

- There is some $K>0$ such that $F V_{2}(n) \leq K n^{2}$ for all $n \in \mathbb{N}$.

$$
\lim _{n \rightarrow \infty} \frac{F V_{3}(n)}{n^{\frac{3}{2}}}=0 .
$$

- There is some $g \in \mathbb{N}$ such that if $S$ is a minimizer 2 -cycle in $X^{2}$, then $S$ is represented by a surface of genus at most $g$.

Then for every $\epsilon>0$ we have

$$
\lim _{n \rightarrow \infty} \frac{F V_{3}(n)}{n^{1+\epsilon}}=0 .
$$

We don't know whether the third condition on the bound of the genus of the minimizers is in fact necessary. It is quite crucial however for our proof. We use it to deduce the fact that the diameter of a minimizer $S$ is of the order of $\sqrt{A(S)}$. This in turn is based on the upper bound of Cheeger constants in terms of genus. So our proof would work as well if we assumed that there is an upper bound for 
the Cheeger constant of minimizers of the form $\frac{c}{\sqrt{A(S)}}$ or if we assumed that there is a lower bound for the diameter of minimizers of the form $\delta \sqrt{A(S)}$. It would be interesting to remove the condition on minimizers even in the case where $X$ is a non-positively curved manifold homeomorphic to $\mathbb{R}^{3}$. We remark that it is not known whether the isoperimetric profile of a non-positively curved manifold homeomorphic to $\mathbb{R}^{n}$ is dominated by the isoperimetric profile of the Euclidean space $\mathbb{E}^{n}$ (this is known however for $n \leq 4$; see [11], 23]).

We are going to prove a somewhat stronger statement that implies Theorem 4.1.

Theorem 4.2. Let $X$ be a simplicial complex such that $H_{1}(X)=H_{2}(X)=0$. Assume that the following hold:

- There is some $K>0$ such that $F V_{2}(n) \leq K n^{2}$ for all $n \in \mathbb{N}$.

- There is some $g \in \mathbb{N}$ such that if $S$ is a minimizer 2 -cycle in $X^{2}$, then $S$ is represented by a surface of genus at most $g$.

Then given $\epsilon>0$ there is a constant $\alpha=\alpha(K, g)>0$ such that the following holds: If there is an $n_{0}$ such that for all $n>n_{0}, F V_{3}(n)<\alpha n^{\frac{3}{2}}$, then

$$
\lim _{n \rightarrow \infty} \frac{F V_{3}(n)}{n^{1+\epsilon}}=0
$$

Proof. In the course of the proof we will need to introduce some new constants; we will indicate the previous constants that the new constants depend on, e.g. for the new constant $c$ we write $c=c(A, B)$ to indicate that $c$ depends on the previous defined constants, $A, B$. It is always possible to give explicit estimates for the constants, but we refrain from doing this as we don't find it instructive.

To show the theorem it is enough to show that for any $\epsilon>0$ there is some $\beta=\beta(\epsilon, K, g)>0$ with the following property: If there is an $n_{0}$ such that for all $n>n_{0}, F V_{3}(n)<\beta n^{\frac{3}{2}}$, then

$$
\limsup _{n \rightarrow \infty} \frac{F V_{3}(n)}{n^{1+\epsilon}} \neq \infty
$$

Indeed we can then take $\alpha(\epsilon)=\beta(\epsilon / 2)$.

We argue by contradiction. The value of $\beta$ will be specified in the course of the proof. So we assume that for some $\epsilon>0$ the following holds:

For any $M>0$ there is some 2-cycle $S$ such that FillV $(S)>M A(S)^{1+\epsilon}$.

Lemma 4.3. There is a $\delta=\delta(\epsilon, g, K)>0$ such that the following holds:

For any $M>0$, if $S$ is a 2-cycle such that FillV $(S)>M A(S)^{1+\epsilon}$, and $A(S)$ is minimal among all cycles for which this inequality holds, then $\operatorname{diam}(S)>\delta \sqrt{A(S)}$.

Proof. We set $n=\sqrt{A(S)}$. As we saw in the proof of Proposition 2.6 there is a decomposition of $S$ in two pieces $S_{1}, S_{2}$ such that:

- $S=S_{1} \cup S_{2}$.

- $S_{1} \cup S_{2}=\partial S_{1} \cap \partial S_{2}$.

- $A\left(S_{i}\right) \geq A(S) / 4, i=1,2$.

- $l\left(S_{1} \cap S_{2}\right) \leq 10^{3} g^{2} n$.

- $S_{1} \cap S_{2}$ has at most $2 g+1$ components.

We claim now that if $p$ is a closed curve on $X^{1}$ of diameter less than $\delta l(p)$, then Fill $A(p) \leq 40 K \delta l(p)^{2}$. 
To see this subdivide $p$ into $r=[1 / \delta]+1$ segments of length at most $\delta l(p)+1$. Let $v_{1}, \ldots, v_{r}$ be the successive endpoints of these segments. We consider geodesic segments $\left[v_{1}, v_{3}\right], \ldots,\left[v_{1}, v_{r-1}\right]$, and we use them to break $p$ into $r-2$ loops each of which has length at most $3 \delta l(p)+3$. Since Fill $A(p)$ is less than or equal to the sum of the areas of these loops we have

$$
\text { Fill } A(p) \leq K([1 / \delta]-1)(3 \delta l(p)+3)^{2} \leq K \frac{1}{\delta}(6 \delta l(p))^{2} \leq 40 K \delta l(p)^{2} .
$$

Assume now that $\operatorname{diam}(S) \leq \delta n$. We will show that this leads to a contradiction if $\delta$ is too small.

Let $c=S_{1} \cap S_{2}$. Since $c$ has at most $2 g+1$ components and $\operatorname{diam}(c) \leq \operatorname{diam}(S) \leq$ $\delta n$, using the above estimate for a single simple closed curve we obtain for $c$ :

$$
\text { Fill } A(c) \leq 40 K(2 g+1) \delta l(c)^{2} \leq 40 K(2 g+1) \delta\left(10^{3} g^{2} n\right)^{2} .
$$

Let $\tilde{S}$ be a 2 -cycle filling $c$ with $A(\tilde{S}) \leq 40 K(2 g+1) \delta l(c)^{2}$. We break $S$ into two 2-cycles using $\tilde{S}$ : $\tilde{S}_{1}=S_{1}+\tilde{S}$ and $\tilde{S}_{2}=S_{2}-\tilde{S}$. We set $\delta^{\prime}=40 K(2 g+1) 10^{6} g^{4} \delta$. So

$$
A(\tilde{S}) \leq \delta^{\prime} A(S) .
$$

If $\delta$ is sufficiently small $A(\tilde{S})$ is smaller than $A\left(S_{1}\right), A\left(S_{2}\right)$. Using the minimality of $S$ we have

$$
\operatorname{FillV}(S) \leq \operatorname{FillV}\left(\tilde{S}_{1}\right)+\operatorname{FillV}\left(\tilde{S}_{2}\right) \leq M A\left(\tilde{S}_{1}\right)^{1+\epsilon}+M A\left(\tilde{S}_{2}\right)^{1+\epsilon} .
$$

Since $A\left(S_{1}\right)$ and $A\left(S_{2}\right)$ are bigger than $A(S) / 4$ there is some $a \in\left[\frac{1}{4}, \frac{3}{4}\right]$ such that $A\left(S_{1}\right)=a A(S)$ and $A\left(S_{2}\right)=(1-a) A(S)$. We have

$$
A\left(\tilde{S}_{1}\right) \leq a A(S)+\delta^{\prime} A(S), A\left(\tilde{S}_{2}\right) \leq(1-a) A(S)+\delta^{\prime} A(S) .
$$

Substituting above we have

$$
\operatorname{FillV}(S) \leq M A(S)^{1+\epsilon}\left[\left(a+\delta^{\prime}\right)^{1+\epsilon}+\left(1-a+\delta^{\prime}\right)^{1+\epsilon}\right] .
$$

Since the function $x^{1+\epsilon}$ is strictly convex $a^{1+\epsilon}+(1-a)^{1+\epsilon}<1$ for all $a \in(0,1)$. It follows that if $\delta^{\prime}$ is small enough $\left(a+\delta^{\prime}\right)^{1+\epsilon}+\left(1-a+\delta^{\prime}\right)^{1+\epsilon}<1$. Clearly one can give an explicit estimate for $\delta^{\prime}$ in terms of $\epsilon$.

Now if

we have

$$
\delta=\frac{\delta^{\prime}}{40 K(2 g+1) 10^{6} g^{4}}
$$

$$
\operatorname{FillV}(S) \leq M A(S)^{1+\epsilon}
$$

which is a contradiction.

We need a technical lemma:

Lemma 4.4. Given $\epsilon>0$ there is some $\lambda \in(0,1 / 2)$ such that for any $x \in(0,1 / 2]$ the following inequality holds:

$$
(x+\lambda x)^{1+\epsilon}+(1-x+\lambda x)^{1+\epsilon}<1 .
$$

Proof. We consider the function

$$
f(x)=1-(x+\lambda x)^{1+\epsilon}-(1-x+\lambda x)^{1+\epsilon} .
$$

We have

$$
f^{\prime}(x)=(1+\epsilon)\left[-(1+\lambda)^{1+\epsilon} x^{\epsilon}+(1-\lambda)(1-x+\lambda x)^{\epsilon}\right] .
$$


We now remark that there is a constant $c>0$ such that if $\lambda<1 / 2$ we have

$$
-(1+\lambda)^{1+\epsilon} x^{\epsilon}+(1-\lambda)(1-x+\lambda x)^{\epsilon}>0, \forall x \in[0, c] .
$$

Since $f(0)=0$ we conclude that $f(x)>0$ for $x \in(0, c]$, if $\lambda<1 / 2$.

Now we remark that the function $x^{1+\epsilon}$ is strictly convex. It follows that $x^{1+\epsilon}+$ $(1-x)^{1+\epsilon}$ restricted on the interval $[c, 1 / 2]$ is strictly smaller than 1 . It follows that there is some $\lambda>0$ such that

$$
(x+\lambda x)^{1+\epsilon}+(1-x+\lambda x)^{1+\epsilon}<1
$$

for all $x \in[c, 1 / 2]$. So there is some $1 / 2>\lambda>0$ such that for any $x \in(0,1 / 2)$ we have

$$
(x+\lambda x)^{1+\epsilon}+(1-x+\lambda x)^{1+\epsilon}<1 .
$$

In what follows, given $M>0$ we consider a 2-cycle $S$ of minimal area such that FillV $(S)>M A(S)^{1+\epsilon}$. Let $R$ be a 3 -chain such that $\partial R=S$ and $V(R)=$ FillV $(S)$. We consider $R$ as a subset of $X$. We fix a vertex $x \in S$, and we consider $B_{i}(x)$ in $X$. Let $R=\sum n_{j} \sigma_{j}$ with $n_{j} \in \mathbb{Z}$. We define $R_{i}$ to be the chain

$$
R_{i}=\sum_{\sigma_{k} \subset B_{i}(x)} n_{k} \sigma_{k} .
$$

We now consider all decompositions of $\partial R_{i}$ as sum of two chains $\partial R_{i}=T_{1}+T_{2}$. We consider the minimal value of $A\left(S-T_{1}\right)$ over all such decompositions. Let $\partial R_{i}=O_{i}+I_{i}$ be a decomposition of $\partial R_{i}$ such that $A\left(S-O_{i}\right)$ attains this minimum.

With this notation we have the following lemma.

Lemma 4.5. There is a $\lambda>0$ such that for any $M>0$, if $S$ is a 2-cycle $S$ such that FillV $(S) \geq M A(S)^{1+\epsilon}$, and which has minimal area, $A(S)$, among all cycles verifying the previous inequality, then the following holds:

$$
A\left(I_{i}\right) \geq \lambda \min \left\{A\left(O_{i}\right), A\left(S-O_{i}\right)\right\}, \quad \forall i \in\left[\frac{\delta n}{4}, \frac{\delta n}{2}\right],
$$

where $\delta$ is given by Lemma 4.3 and we denote as before $n=\sqrt{A(S)}$.

Proof. Let $\lambda$ be as in Lemma 4.4. We argue by contradiction, i.e. we assume that the inequality of the lemma does not hold for some $i$. We consider the 2-cycles $\partial R_{i}$ and $S-\partial R_{i}$. We remark that $A\left(\partial R_{i}\right)$ and $A\left(S-\partial R_{i}\right)$ are both smaller than $A(S)$. By our assumption on $S$ we have the inequalities

$$
\begin{aligned}
\text { FillV }\left(\partial R_{i}\right) & <M A\left(\partial R_{i}\right)^{1+\epsilon}, \\
\text { FillV }\left(S-\partial R_{i}\right) & <M A\left(S-\partial R_{i}\right)^{1+\epsilon} .
\end{aligned}
$$

We also have

$$
\operatorname{FillV}(S) \leq \operatorname{FillV}\left(\partial R_{i}\right)+\operatorname{FillV}\left(S-\partial R_{i}\right) .
$$

Hence

$$
\text { FillV }(S)<M\left[A\left(\partial R_{i}\right)^{1+\epsilon}+A\left(S-\partial R_{i}\right)^{1+\epsilon}\right]
$$

Now $A\left(\partial R_{i}\right)=A\left(O_{i}\right)+A\left(I_{i}\right)$ and $A\left(S-\partial R_{i}\right)=A\left(I_{i}\right)+A\left(S-O_{i}\right)$. Let

$$
m=\max \left\{A\left(O_{i}\right), A\left(S-O_{i}\right)\right\} \text {. }
$$

We set

$$
x=\frac{m}{A(S)} .
$$


From inequality (1) and from our assumption we obtain

$$
\text { FillV }(S)<M A(S)^{1+\epsilon}\left[(x+\lambda x)^{1+\epsilon}+(1-x+\lambda x)^{1+\epsilon}\right]<M A(S)^{1+\epsilon},
$$

where the last inequality follows from Lemma 4.4. This is clearly a contradiction.

Lemma 4.6. Let $\mu>0, a \in \mathbb{N}, a>3$ and let $f(i) \in \mathbb{R}$ be a sequence such that $f(i)>\mu a$ for all $i \in \mathbb{N} \cap[a, 2 a]$. Let

$$
F(s)=\sum_{i=a}^{s} f(i)
$$

and assume that $F(2 a)<\mu a^{3}$. Then for some $r$ we have

$$
F(r)>\frac{1}{8 \sqrt{3 \mu}} f(r)^{\frac{3}{2}} .
$$

Proof. We consider the sequence

$$
g(i)=3 \mu i^{2}, \quad i=1,2, \ldots, a .
$$

We have $f(a)>g(1)$ and

$$
\sum_{i=1}^{a} g(i)>\sum_{i=a}^{2 a} f(i)
$$

We consider the smallest $r \geq a$ for which

$$
f(r)<g(r-a+1) .
$$

We then have

$$
F(r)>F(r-1)=\sum_{i=a}^{r-1} f(i)>\sum_{i=1}^{r-a} g(i) .
$$

We set $s=r-a+1$, and we have

$$
\sum_{i=1}^{r-a} g(i)=3 \mu \frac{(s-1) s(2 s-1)}{6} .
$$

On the other hand

$$
f(r)<g(s)=3 \mu s^{2}
$$

Since $s \geq 2$ we have

$$
3 \mu \frac{(s-1) s(2 s-1)}{6} \geq \frac{1}{8 \sqrt{3 \mu}}\left(3 \mu s^{2}\right)^{\frac{3}{2}}>\frac{1}{8 \sqrt{3 \mu}} f(r)^{\frac{3}{2}},
$$

so

$$
F(r)>\frac{1}{8 \sqrt{3 \mu}} f(r)^{\frac{3}{2}} .
$$

We now take $M=M\left(n_{0}\right)$ 'sufficiently big', and we consider a 2-cycle $S$ of minimal $A(S)$ such that $F i l l V(S)>M A(S)^{1+\epsilon}$. We will explain how we choose $M$ at the relevant point of the proof.

Let $R$ be a 3-chain such that $\partial R=S$ and $V(R)=F i l l V(S)$. We set $n=\sqrt{A(S)}$. 
Let $\delta$ be as in Lemma 4.3. We fix a vertex $x \in S$, and we consider $B_{i}(x) \cap S$ and $B_{i}(x) \cap R$ for $\delta n / 4 \leq i \leq \delta n / 2$. We define $R_{i}, O_{i}, I_{i}$ as above. We remark now that the following inequalities hold:

$$
\begin{gathered}
A\left(O_{i}\right) \geq 2 i \geq \delta n / 2, \\
A\left(S-O_{i}\right) \geq \delta n / 2, \\
V\left(R_{i}-R_{i-1}\right) \geq A\left(I_{i}\right) / 3 \geq \lambda \delta n / 6,
\end{gathered}
$$

where $\lambda$ is the constant provided by lemma 4.5 and the last inequality follows from the same lemma.

We now consider the finite sequence

$$
f(i)=A\left(I_{i}\right), i \in[\delta n / 4, \delta n / 2] .
$$

We may choose $M$ so that $\delta n / 4>3$. We may also assume that $\delta$ is such that $\delta n / 4 \in \mathbb{N}$ (we just replace $\delta$ by $\delta^{\prime}$ so that $\delta^{\prime} n / 4=[\delta n / 4]$ ).

We set $a=\delta n / 4$, and we remark that $f(i) \geq \frac{\lambda}{2} a$ for all $i \in[\delta n / 4, \delta n / 2]$. We may choose $\beta$ so that $3 \beta<\frac{\lambda}{2}$. Since $V(R)<\beta n^{3}$ we have

$$
\sum_{i=a}^{2 a} f(i) \leq V(R) \leq 3 \beta n^{3}<\frac{\lambda}{2} n^{3}
$$

by our assumption and the inequality $(*)$.

Applying Lemma 4.6 to $f$ with $\mu=\frac{\lambda}{2}$ we conclude that there is some $r \in$ $[\delta n / 4, \delta n / 2]$ such that

We also have the inequality

$$
\sum_{i=a}^{r} f(i)>\frac{1}{8 \sqrt{3 \mu}} f(r)^{\frac{3}{2}}
$$

$$
V\left(R_{r}\right) \geq \sum_{j=\delta n / 4}^{r} f(j) / 3 \geq \frac{1}{24 \sqrt{3 \mu}} f(r)^{\frac{3}{2}} .
$$

From Lemma 4.5

$$
f(r) \geq \lambda \min \left\{A\left(O_{r}\right), A\left(S-O_{r}\right)\right\} .
$$

We now distinguish two cases:

Case 1. $f(r) \geq \lambda A\left(O_{r}\right)$. In this case

$$
A\left(\partial R_{r}\right)=A\left(O_{r}\right)+A\left(I_{r}\right) \leq\left(1+\frac{1}{\lambda}\right) f(r),
$$

while

So if

$$
V\left(R_{r}\right) \geq \frac{1}{24 \sqrt{3 \mu}} f(r)^{\frac{3}{2}} .
$$

$$
\beta \leq \min \left(\frac{1}{24 \sqrt{3 \mu}\left(1+\frac{1}{\lambda}\right)^{\frac{3}{2}}}, \frac{\lambda}{6}\right)
$$

and $M$ is big enough so that $A\left(\partial R_{r}\right)>n_{0}$, we have

$$
V\left(R_{r}\right) \geq \beta A\left(\partial R_{r}\right)^{\frac{3}{2}},
$$

which contradicts our assumption.

Case 2. $f(r)<\lambda A\left(O_{r}\right)$. Then $f(r) \geq A\left(S-O_{r}\right)$ so $A\left(S-O_{r}\right) \leq A\left(O_{r}\right)$. In this case we pick $y \in S$ with $d(x, y)=\operatorname{diam} S^{1}$ and we repeat the construction 
considering $B_{y}(i)$ instead of $B_{x}(i)$. We obtain a 3-chain as before which we denote $R_{r^{\prime}}^{\prime}$. Since $\partial R_{r^{\prime}}^{\prime} \cap S \subset A\left(S-O_{r}\right)$ we obtain a contradiction from $\partial R_{r^{\prime}}^{\prime}$ as in Case 1.

Remark 6 . The assumption that $F V_{2}(n)$ is bounded by a quadratic function does not play an essential role in the proof above. One may substitute this by $F V_{2}(n) \leq$ $K n^{r}$ for some $K>0, r>2$ and change the conclusion to: If

$$
\lim _{n \rightarrow \infty} \frac{F V_{3}(n)}{n^{\frac{2 r-1}{2 r-2}}}=0
$$

then

$$
\lim _{n \rightarrow \infty} \frac{F V_{3}(n)}{n^{1+\epsilon}}=0
$$

for any $\epsilon>0$. This shows that there is some relationship between $F V_{2}(n)$ and $F V_{3}(n)$, always of course under the assumption of the bound on the genus of minimizers.

\section{ACKNOWLEDGMENTS}

The author would like to thank the referee for many corrections and suggestions that helped improve this paper.

\section{REFERENCES}

[1] F. J. Almgren, Jr., An isoperimetric inequality, Proc. Amer. Math. Soc. 15 (1964), 284-285. MR0159925 (28:3141)

[2] A. S. Besicovitch, On two problems of Loewner, J. London Math. Soc. 27 (1952), 141-144. MR0047126 (13:831d)

[3] M. Bonk, A. Eremenko, Uniformly hyperbolic surfaces, Indiana Univ. Math. J. 49 (2000), no. 1, 61-80. MR1777037 (2001g:53120)

[4] N. Brady, M. R. Bridson, There is only one gap in the isoperimetric spectrum, Geom. Funct. Anal. 10 (2000), no. 5, 1053-1070. MR.1800063 (2001j:20046)

[5] N. Brady, M. R. Bridson, M. Forester, K. Shankar, Snowflake groups, Perron-Frobenius eigenvalues, and isoperimetric spectra, preprint.

[6] M. R. Bridson, Fractional isoperimetric inequalities and subgroup distortion, J. Amer. Math. Soc. 12 (1999), no. 4, 1103-1118. MR.1678924 (2001a:20062)

[7] I. Benjamini, J. Cao, A new isoperimetric theorem for surfaces of variable curvature, Duke Math. J. 85 (1996), 359-396. MR1417620 (97m:58046)

[8] B. H. Bowditch, A short proof that a subquadratic isoperimetric inequality implies a linear one, Michigan Math. J. 42 (1995), no. 1, 103-107. MR1322192 (96b:20046)

[9] J. Burillo, J. Taback, Equivalence of geometric and combinatorial Dehn functions, New York J. Math. 8 (2002), 169-179. MR.1934388 (2004c:20070)

[10] M. Coornaert, T. Delzant, A. Papadopoulos, Geometrie et theorie des groupes, Lecture Notes in Mathematics, 1441, Springer-Verlag, Berlin, 1990. x+165 pp. MR.1075994 (92f:57003)

[11] C. B. Croke, A sharp four-dimensional isoperimetric inequality., Comment. Math. Helv. 59 (1984), no. 2, 187-192. MR749103 (85f:53060)

[12] C. Drutu, Cones asymptotiques et invariants de quasi-isometrie pour des espaces metriques hyperboliques, Ann. Inst. Fourier (Grenoble) 51 (2001), no. 1, 81-97. MR1821069 (2002h:53069)

[13] D. B. A. Epstein, J. W. Cannon, D. F. Holt, S. V. F. Levy, M. S. Paterson, W. P. Thurston, Word processing in groups, Jones and Bartlett Publishers, Boston, MA, 1992. xii+330 pp. MR.1161694 (93i:20036)

[14] S. M. Gersten, Subgroups of word hyperbolic groups in dimension 2, J. London Math. Soc. (2) 54 (1996), no. 2, 261-283. MR1405055 (97e:20058)

[15] J. R. Gilbert, J. P. Hutchinson, R. E. Tarjan, A separator theorem for graphs of bounded genus J. Algorithms 5 (1984), 391-407. MR756165 (86h:68145) 
[16] R. Grimaldi, P. Pansu, Remplissage et surfaces de revolution, J. Math. Pures Appl. (9) 82 (2003), no. 8, 1005-1046. MR2005203 (2004f:53032)

[17] M. Gromov, Filling Riemannian manifolds J. Differential Geom. 18 (1983), no. 1, 1-147. MR697984 (85h:53029)

[18] M. Gromov, Hyperbolic groups, Essays in group theory (S. M. Gersten, ed.), MSRI Publ. 8, Springer-Verlag, 1987, 75-263. MR919829 (89e:20070)

[19] M. Gromov, Asymptotic invariants of infinite groups in 'Geometric group theory' (G. Niblo, M. Roller, Eds.), LMS Lecture Notes, vol. 182, Cambridge Univ. Press, 1993. MR.1253544 (95m:20041)

[20] M. Gromov, Metric structures for Riemannian and non-Riemannian spaces, with appendices by M. Katz, P. Pansu and S. Semmes, Progress in Mathematics, 152, Birkhauser Boston, Inc., Boston, MA, 1999. xx+585 pp. MR1699320(2000d:53065)

[21] A. Hatcher, Algebraic topology, Cambridge University Press, Cambridge, 2002. xii+544 pp. MR1867354 (2002k:55001)

[22] J. Hersch, Quatre proprietes isoperimetriques de membranes spheriques homogenes, C.R. Acad. Sci. Paris 270 (1970), 1645-1648. MR0292357 (45:1444)

[23] B. Kleiner, An isoperimetric comparison theorem, Invent. Math. 108 (1992), no. 1, 37-47. MR1156385 (92m:53056)

[24] N. Korevaar, Upper bounds for eigenvalues of conformal metrics, J. Diff. Geom. 37 (1993), 73-93. MR.1198600 (94d:58153)

[25] R. J. Lipton, R. E. Tarjan, Applications of a planar separator theorem, SIAM J. Comput. 9 (1980), 615-627. MR584516 (82e:68067)

[26] F. Morgan, M. Hutchings, H. Howards, The isoperimetric problem on surfaces of revolution of decreasing Gauss curvature, Trans. Amer. Math. Soc. 352 (2000), no. 11, 4889-4909. MR:1661278 (2001b:58024)

[27] A. Yu. Olshanski, Hyperbolicity of groups with subquadratic isoperimetric inequality, Internat. J. Algebra Comput. 1 (1991), no. 3, 281-289. MR.1148230 (93d:20067)

[28] P. Pansu, Une inégalité isopérimétrique pour le groupe d'Heisenberg, C.R. Acad. Sci. Paris 295 (1982), 127-131. MR676380 (85b:53044)

[29] P. Papasoglu, An algorithm detecting hyperbolicity, Geometric and computational perspectives on infinite groups, 193-200, DIMACS Ser. Discrete Math. Theoret. Comput. Sci., 25, AMS, Providence, RI, 1996. MR 1364185 (96k:20075)

[30] P. Papasoglu, Isodiametric and isoperimetric inequalities for complexes and groups, J. London Math. Soc. (2) 62 (2000), no. 1, 97-106. MR.1771853 (2001h:20052)

[31] L. Polterovich, J-C. Sikorav, A linear isoperimetric inequality for the punctured Euclidean plane, preprint, arXive math.GR/0106216.

[32] M. Ritoré, The isoperimetric problem in complete surfaces of nonnegative curvature, J. Geom. Anal. 11 (2001), no. 3, 509-517. MR.1857855 (2002f:53109)

[33] M. Ritoré, Constant geodesic curvature curves and isoperimetric domains in rotationally symmetric surfaces, Comm. Anal. Geom. 9 (2001), no. 5, 1093-1138. MR1883725 (2003a:53018)

[34] M. V. Sapir, J-C. Birget, E. Rips, Isoperimetric and isodiametric functions of groups, Ann. of Math. (2) 156 (2002), no. 2, 345-466. MR.1933723 (2005b:20077a)

[35] P. Topping, Mean curvature flow and geometric inequalities, J. Reine Angew. Math. 503 (1998), 47-61. MR:1650335 (99m:53080)

[36] X. Wang, S. J. Pride, Second order Dehn functions and HNN-extensions, J. Austral. Math. Soc. Ser. A 67 (1999), no. 2, 272-288. MR.1717418 (2002k:20076)

[37] S. Wenger, Gromov hyperbolic spaces and the sharp isoperimetric constant, Invent. Math. 171 (2008), no. 1, 227-255. MR2358060

[38] S. Wenger, Isoperimetric inequalities and the asymptotic rank of metric spaces, preprint.

[39] P. Yang, S. T. Yau, Eigenvalues of the Laplacian of compact Riemann surfaces and minimal submanifolds, Ann. Scuola Norm. Sup. Pisa Cl. Sci. (4) 7 (1980), 55-63. MR577325 (81m:58084)

Department of Mathematics, University of Athens, Athens 157 84, Greece

E-mail address: panos@math.uoa.gr 